\title{
Lycorine protects cartilage through suppressing the expression of matrix metalloprotenases in rat chondrocytes and in a mouse osteoarthritis model
}

\author{
SHUAI CHEN ${ }^{1,2}$, XIANG-QIAN FANG $^{1,2}$, JIAN-FENG ZHANG $^{1,2}$, YAN MA $^{1,2}$, XIAO-ZHEN TANG $^{1,2}$, \\ ZHI-JIE ZHOU ${ }^{1,2}$, JI-YING WANG ${ }^{1,2}$, AN QIN $^{3}$ and SHUN-WU FAN ${ }^{1,2}$ \\ ${ }^{1}$ Department of Orthopaedics; ${ }^{2}$ Key Laboratory of Biotherapy of Zhejiang, Sir Run Run Shaw Hospital, \\ School of Medicine, Zhejiang University, Hangzhou, Zhejiang 310016; ${ }^{3}$ Department of Orthopaedics, \\ Shanghai Key Laboratory of Orthopaedic Implant, Shanghai Ninth People's Hospital, \\ Shanghai Jiaotong University School of Medicine, Shanghai 200011, P.R. China
}

Received May 18, 2015; Accepted March 21, 2016

DOI: $10.3892 / \mathrm{mmr} .2016 .5594$

\begin{abstract}
Extracellular matrix (ECM) degrading enzymes, including matrix metalloproteinases (MMPs), are critical for cartilage destruction in the progression of osteoarthritis (OA). Thus, identifying novel drugs, which suppress the synthesis of MMPs may facilitate the treatment of OA. The cytotoxicity of lycorine was determined using a CCK8 assay. The effects of lycorine on IL-1 $\beta$-induced upregulation of MMPs and activation of mitogen-activated protein kinase pathways were detected by western blot analysis and reverse transcription-quantitative polymerase chain reaction. Hematoxylin and eosin staining and Safranin O staining were used to evaluate the effect of lycorine in a mouse anterior cruciate ligament transection model. In the present study, it was demonstrated for the first time, to the best of our knowledge, that lycorine (LY) suppressed interleukin-1 $\beta$ (IL-1 $\beta$ )-induced synthesis of MMP-3 and MMP-13 in vitro. Molecular analysis revealed that LY abrogated the phosphorylation of c-Jun N-terminal kinase (JNK) and the activation of the nuclear factor $(\mathrm{NF})-\kappa \mathrm{B}$ signaling pathway caused by IL-1 $\beta$ stimulation. In addition, in vivo experiments in a mouse anterior cruciate ligament transection model confirmed the protective role of LY on cartilage. Taken together, the data obtained in the present
\end{abstract}

Correspondence to: Dr Shun-Wu Fan, Department of Orthopaedics, Sir Run Run Shaw Hospital, School of Medicine, Zhejiang University, 3 East Qinchun Road, Hangzhou, Zhejiang 310016, P.R. China

E-mail: srrshspine@163.com

Dr An Qin, Department of Orthopaedics, Shanghai Key Laboratory of Orthopaedic Implant, Shanghai Ninth People's Hospital, Shanghai Jiaotong University School of Medicine, 639 Zhizaoju Road, Shanghai 200011, P.R. China

E-mail: dr_qinan@163.com

Key words: cartilage, matrix metalloproteinases, nuclear factor- $\kappa \mathrm{B}$, lycorine, mitogen-activated protein kinase study demonstrated that LY suppressed the IL-1 $\beta$-induced expression of MMP-3 and MMP-13 through inhibition of the JNK and NF- $\kappa \mathrm{B}$ pathways, suggesting that LY may be used as a potential drug for the treatment of OA.

\section{Introduction}

Osteoarthritis (OA) is the most common type of joint disease, and can cause chronic pain and disability, particularly in the elderly population (1). Progressive cartilage destruction is one of the most important characteristics of OA (2). Cartilage consists of chondrocytes and extracellular matrix (ECM). Although the etiology of cartilage impairment remains to be fully elucidated, imbalance in the anabolism and catabolism of cartilage ECM is considered to be one of the predominant mechanisms of cartilage degradation. The ECM of cartilage is predominantly composed of type II collagen and aggrecan (3). Under normal conditions, the synthesis and hydrolysis of these ECM proteins are in dynamic balance. However, when OA begins to develop, the levels of inflammatory cytokines, including interleukin-1 $\beta$ (IL-1 $\beta$ ) and tumor necrosis factor- $\alpha$ (TNF- $\alpha$ ) increase, leading to the upregulation of matrix degrading enzymes, including matrix metalloproteinases (MMPs) and a disintergrin and metalloproteinases with thrombospondin motifs (ADAMTSs) $(4,5)$. This occurs through the nuclear factor $(\mathrm{NF})-\kappa \mathrm{B}$ and mitogen-activated protein kinase (MAPK) signaling pathways (6). MMPs are metal-dependent proteases, which are capable of degrading components of the connective tissue ECM (7) and are major proteases in cartilage degradation. MMP-3 and MMP-13 are among the most important collagenases and aggrecanases during the progression of OA $(8,9)$, and increased expression levels of these catabolic enzymes are considered the hallmark of cartilage degeneration. Thus, the inhibition of MMPs may be a valuable treatment strategy for OA.

An increasing number of natural compounds have been shown to protect articular cartilage (10-12). Lycorine (LY) is a natural alkaloid, which is extracted from flowers and bulbs of Amaryllidaceae species (13). In previous studies, 
LY was found to exhibit potential antitumor effects against ovarian cancer, myeloid leukemia and melanoma with low toxicity (14-16). In addition, LY has been shown to suppress lipopolysaccharide-induced inflammation via inhibition of the MAPK signaling pathway in a macrophage cell line (17). As MMPs are induced by proinflammatory cytokines through the NF- $\mathrm{BB}$ and MAPK signaling pathways, LY may be involved in reducing the expression of MMP and preventing cartilage degeneration. The present study was designed to examine the protective role of LY and its underlying mechanism of action in cultured rat articular chondrocytes and in a mouse anterior cruciate ligament transection (ACLT) model. An understanding of the effects of lycorine on cartilage and its associated mechanism may develop a novel, suitable therapy to treat osteoarthritis and other cartilage-associated diseases.

\section{Materials and methods}

Ethical approval. All experiments performed in the present study were approved by the Ethics Committee of Sir Run Run Shaw Hospital (Hangzhou, China).

Primary rat articular chondrocyte culture. The animal experiments in the present study were performed in accordance with the National Institutes of Health Guide for the Care and Use of Laboratory Animals (18). The present study was approved by the ethics committee of Sir Run Run Shaw Hospital, (Hangzhou, China). Sprague-Dawley rats (male; weight, $\sim 200 \mathrm{~g}$; age, 2 months) were supplied by the laboratory animal center of Zhejiang University and were housed at $\sim 24^{\circ} \mathrm{C}$, with free access to food and water were used in the current study. The bilateral knee joints of rats ( $n=3 /$ experiment) were separated and finely diced into small pieces measuring no more than $1 \mathrm{~mm}^{3}$. The tissues were treated with $0.2 \%$ collagenase (Sigma-Aldrich, St. Louis, MO, USA) for $4 \mathrm{~h}$ at $37^{\circ} \mathrm{C}$. Following centrifugation at $800 \mathrm{x} \mathrm{g}$ for $5 \mathrm{~min}$ at $37^{\circ} \mathrm{C}$, the supernatant was placed in Dulbecco's modified Eagle's medium (Haining Jinuo Biomedical Technology Co., Ltd., Hangzhou, China) supplemented with $10 \%$ fetal bovine serum (Gibco; Thermo Fisher Scientific, Inc., Waltham, MA, USA) in a humidified atmosphere containing $5 \% \mathrm{CO}_{2}$ at $37^{\circ} \mathrm{C}$. To sacrifice the rats at 4 weeks following ACLT surgery, they were administered $5 \mathrm{ml}$ $4 \%$ chloral hydrate by intraperitoneal injection.

Cell viability assay. The cytotoxic effects of LY in the rat chondrocytes were determined using a Cell Counting Kit-8 (CCK-8) assay (Dojindo, Molecular Technologies, Inc. Kumamoto, Japan). The chondrocytes were plated in 96-well plates at a density of $1 \times 10^{4}$ cells/well in triplicate. The cells were then treated with different concentrations of LY $(0,0.06,0.125,0.25$, $0.5,1,2,4,8$ or $16 \mathrm{Mm}$; Sigma-Aldrich) for 24 or $48 \mathrm{~h}$ at $37^{\circ} \mathrm{C}$. Subsequently, $10 \mu \mathrm{l}$ of CCK- 8 buffer was added to each well, and the plates were incubated for an additional $2 \mathrm{~h}$ at $37^{\circ} \mathrm{C}$. The absorbance was measured at a wavelength of $450 \mathrm{~nm}(650 \mathrm{~nm}$ reference) using an ELX800 absorbance microplate reader (Bio-Tek Instruments, Inc., Winooski, VT, USA).

RNA extraction and reverse transcription-quantitative polymerase chain reaction $(R T-q P C R)$ analysis. The primary rat chondrocytes were seeded into 6 -well plates at a density of $10 \times 10^{4}$ cells/well and treated with $10 \mathrm{ng} / \mathrm{ml}$ IL-1 $\beta$ (Sigma-Aldrich) and $0,0.05,0.1,0.2$ or $0.4 \mu \mathrm{M} L Y$ for 24 h. Total RNA was extracted using an RNeasy Mini kit (Qiagen, Valencia, CA, USA). Complementary DNA (cDNA) was synthesized using $0.5 \mu \mathrm{g}$ of RNA from each sample, $2 \mu \mathrm{l}$ of $5 \mathrm{X}$ PrimeScript RT Master mix (Takara Bio, Inc., Otsu, Japan), and $4 \mu \mathrm{l}$ RNase-free $\mathrm{dH}_{2} \mathrm{O}$ in a total volume of $10 \mu \mathrm{l}$. RT-qPCR was performed on an ABI Prism 7500 (Applied Biosystems; Thermo Fisher Scientific, Inc.) using SsoFast EvaGreen supermix (Bio-Rad Laboratories, Inc., Hercules, CA, USA). The total volume $(20 \mu \mathrm{l})$ of each qPCR reaction contained $10 \mu \mathrm{l}$ SsoFast EvaGreen supermix, $7 \mu 1$ $\mathrm{ddH}_{2} \mathrm{O}, 2 \mu \mathrm{l} \mathrm{cDNA}$ and $10 \mu \mathrm{M}$ of each of the forward and reverse primers (Sangon Biotech Co., Ltd., Shanghai, China). The RT-qPCR reaction settings were as follows: $95^{\circ} \mathrm{C}$ for $10 \mathrm{~min}$ (activation), 40 cycles of $95^{\circ} \mathrm{C}$ for $10 \mathrm{sec}, 60^{\circ} \mathrm{C}$ for $20 \mathrm{sec} 72^{\circ} \mathrm{C}$ for $20 \mathrm{sec}$ (amplification) and $72^{\circ} \mathrm{C}$ for $1 \mathrm{~min}$ (final extension), as previously described (19). The quantity of each target was normalized to $\beta$-actin and calculated using the $2^{-\Delta \Delta \mathrm{Cq}}$ method (20). The mouse primer sequences were as follows:

$\beta$-actin, forward 5'-CCTCTATGCCAACACAGT-3' and reverse 5'-AGCCACCAATCCACACAG-3'; MMP-3, forward 5'-TTGTCCTTCGATGCAGTCAG-3' and reverse 5'-AGA CGGCCAAAATGAAGAGA-3'; MMP-13, forward 5'-AGG CCTTCAGAAAAGCCTTC-3' and reverse 5'-GAGCTGCTT GTCCAGGTTTC-3'

Western blotting. The primary rat chondrocytes were seeded into 6 -well plates at a density of $10 \times 10^{4}$ cells/well. To investigate the effects of IL-1 $\beta$ and LY on the expression of MMPs, the cells were treated with IL-1 $\beta(10 \mathrm{ng} / \mathrm{ml})$ with or without $\operatorname{LY}(0,0.05$ or $0.4 \mu \mathrm{M})$ for $48 \mathrm{~h}$ at $37^{\circ} \mathrm{C}$. To investigate the effects of IL-1 $\beta$ and LY on the activation of the signaling pathways, the cells were pre-treated with or without $0.4 \mu \mathrm{M} \mathrm{LY}$ for $2 \mathrm{~h}$, and then stimulated with IL-1 $\beta(10 \mathrm{ng} / \mathrm{ml})$ for $0,5,10,20,30$ or $60 \mathrm{~min}$ at $37^{\circ} \mathrm{C}$. Total protein was extracted from the cultured cells using radioimmunoprecipitation assay lysis buffer (Sigma-Aldrich). The lysates were centrifuged at $12,000 \mathrm{x}$ g for $15 \mathrm{~min}$ at $4^{\circ} \mathrm{C}$, and the supernatants were collected. Proteins were quantified using a BCA kit (Bio-Rad Laboratories, Inc.). The mean concentration of total protein was $\sim 1 \mathrm{mg} / \mathrm{ml}$ in cell lysate and $20 \mu \mathrm{l}$ of cell lysate was loaded on the SDS-PAGE gels. The proteins were resolved by $10 \%$ SDS-PAGE and transferred onto polyvinylidene fluoride membranes by electroblotting (Bio-Rad Laboratories, Inc.). The membranes were blocked in 5\% nonfat dry milk in Tris-buffered saline with Tween 20, containing $50 \mathrm{mM}$ Tris (pH 7.6), $150 \mathrm{mM} \mathrm{NaCl}$ and $0.1 \%$ Tween 20, at room temperature for $1 \mathrm{~h}$. The membranes were then incubated with antibodies purchased from Santa Cruz Biotechnology, Inc., (Dallas, TX, USA) including, MMP-3 (cat no. sc-30070), MMP-13 (cat no. sc-30073), extracellular signal-regulated kinase (ERK; cat no. sc-154), c-Jun N-terminal kinase (JNK; cat no. sc-571), P38 (cat no. sc-535), inhibitor of NF- $\kappa \mathrm{B}(\mathrm{I} \kappa \mathrm{B})-\alpha$ (cat no. sc-847), phosphorylated (p)-ERK (cat no. sc-7383) (Thr202/Tyr204), p-JNK (cat no. sc-6254) (Thr183/Tyr185) and p-p38 (cat no. sc-7973) (Thr180/Tyr182) overnight at $4^{\circ} \mathrm{C}$. All antibodies used were polyclonal, raised in rabbit and rat was the target-species. 
The primary antibodies were used at a dilution of 1:1,000 and the secondary antibody were diluted 1:2,000 and membranes were incubate with the respective secondary antibody for $2 \mathrm{~h}$ at room temperature. Protein bands were developed using a horseradish peroxidase-conjugated goat anti-rabbit immunoglobulin G antibody (Abcam, Cambridge, MA, USA) and were detected using electrochemical luminescence reagent (EMD Millipore, Billerica, MA, USA). The protein bands were visualized using the LAS-4000 Science Imaging System (Fujifilm, Tokyo, Japan).

Mouse ACLT model. A total of 18 8-week-old C57BL/6 mice were randomized into three groups ( $\mathrm{n}=6$ per group) as follows: Sham group, mice subjected to sham surgery+phosphate-buffered saline (PBS); Vehicle group, mice subjected to ACLT and PBS, and LY group, mice subjected to ACLT and $2.5 \mathrm{mg} / \mathrm{kg}$ LY. The ACLT and sham surgical procedures were performed on the right knees of the mice. Animals were anesthetized with $4 \%$ chloral hydrate with the dose of $10 \mathrm{ml} / \mathrm{Kg}$. The operation were performed on the right knees. An incision was formed in the skin laterally to the knee in the ACLT and sham groups. ACL was transected using a surgical scalpel after the joint capsules were opened in ACLT group. In the LY group, $2.5 \mathrm{mg} / \mathrm{kg}$ LY dissolved in $50 \mu \mathrm{l}$ PBS was injected intraperitoneally every other day for 4 weeks. In the Sham and Vehicle groups, the same volume of PBS was injected. All animals were sacrificed 4 weeks following surgery. The right knee joints were fixed in $4 \%$ paraformaldehyde (Sigma-Aldrich) for histological analysis.

Histological analysis. The fixed mouse joints were decalcified in $10 \%$ EDTA for 3 weeks and then embedded in paraffin. In each specimen, three serial sections (4- $\mu \mathrm{m}$ thick) embedded in paraffin were cut using a Leica RM2235 microtome (Leica Microsystems GmbH, Wetzlar, Germany). To observe cell density and morphology, matrix degeneration and proteoglycan content, the sections were stained with hematoxylin and eosin (H\&E; Sigma-Aldrich) and safranin O (SO)-fast green (Sigma-Aldrich). The specimens were examined and images were captured using a high-quality Nikon AZ100, multi-purpose zoom microscope.

Statistical analysis. The data are expressed as the mean \pm standard error of the mean. The results were analyzed using SPSS software for Windows, version 16.0 (SPSS, Inc., Chicago, IL, USA). Student's $t$-test was used to compare between two groups. $\mathrm{P}<0.05$ was considered to indicate a statistically significant difference. Grey level analysis was performed using Image J software (version 1.48; National Institutes of Health, Bethesda, MD, USA)

\section{Results}

Effect of LY on cell viability in rat articular chondrocytes. The cell viability of the chondrocytes treated with LY was assessed to determine which concentrations were not cytotoxic using a CCK-8 assay. The cells were treated with LY at concentrations ranging between 0 and $16 \mu \mathrm{M}$ for 24 and 48 h. As shown in Fig. 1, cell viability was unaffected by LY at concentrations $<0.5 \mu \mathrm{M}$, which indicated that these
A

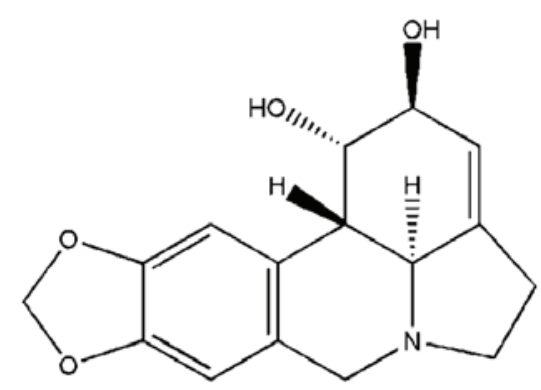

B $24 \mathrm{~h}$

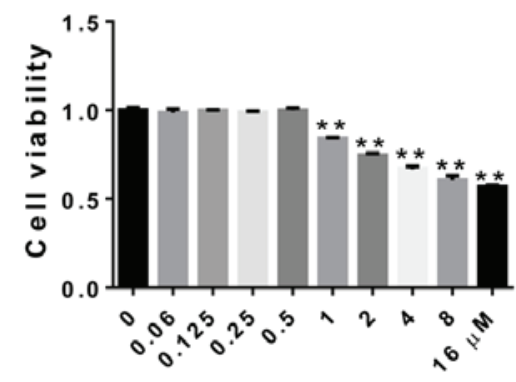

C $48 \mathrm{~h}$

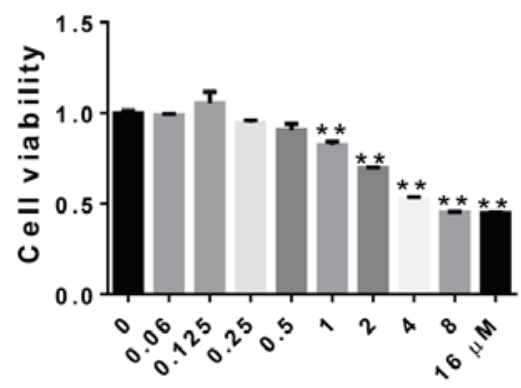

Figure 1. Effect of LY on cell viability in rat articular chondrocytes. (A) Structure of LY. Cell viability of the LY-treated rat articular chondrocytes was determined using a Cell Counting Kit- 8 assay at (B) 24 and (C) 48 h Data are presented as the mean \pm standard error of the mean. $\left({ }^{* *} \mathrm{P}<0.01 \mathrm{vs}\right.$. control group). LY, lycorine.

doses did not have a cytotoxic effect on the rat articular chondrocytes.

$L Y$ inhibits $I L-1 \beta$-induced expression of MMPs in rat articular chondrocytes. To determine the effect of LY on the catabolic activities of chondrocytes, the rat articular chondrocytes were treated with 0-0.4 $\mu \mathrm{M}$ LY and the inflammatory cytokine, IL-1 $\beta$, for 24 or $48 \mathrm{~h}$. The expression levels of MMP-3 and MMP-13 were analyzed using RT-qPCR and Western blotting. Treatment with $10 \mathrm{ng} / \mathrm{ml} \mathrm{IL}-1 \beta$ resulted in upregulation of the mRNA levels of MMP-3 and MMP-13 (Fig. 2A and B). However, the administration of LY led to the downregulation of the expression levels of MMP-3 and MMP-13, which occurred in a dose-dependent manner. The transcriptional levels of MMP-3 and MMP-13 were significantly suppressed by 0.05 and $0.1 \mu \mathrm{M} \mathrm{LY}$, respectively. The levels of MMPs in the groups treated with higher doses of LY $(0.2$ and $0.4 \mu \mathrm{M})$ 
A

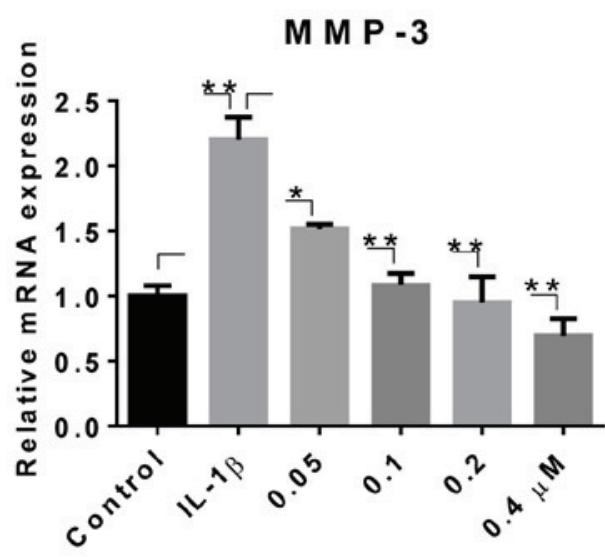

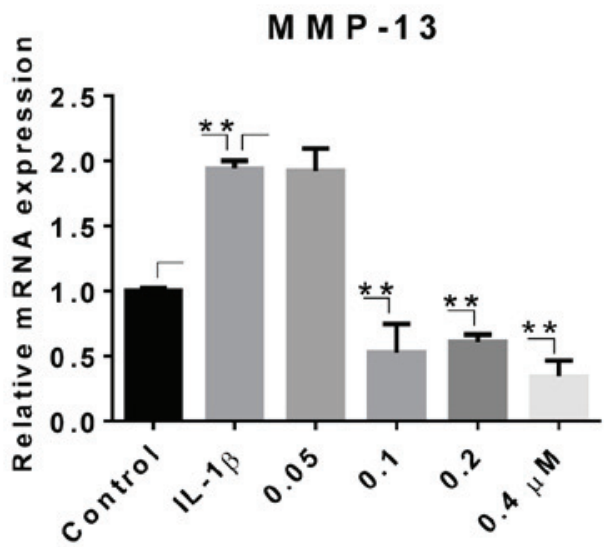

C

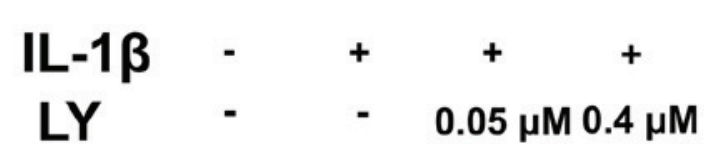

MMP-3

MMP-13

\section{$\beta$-actin}

D

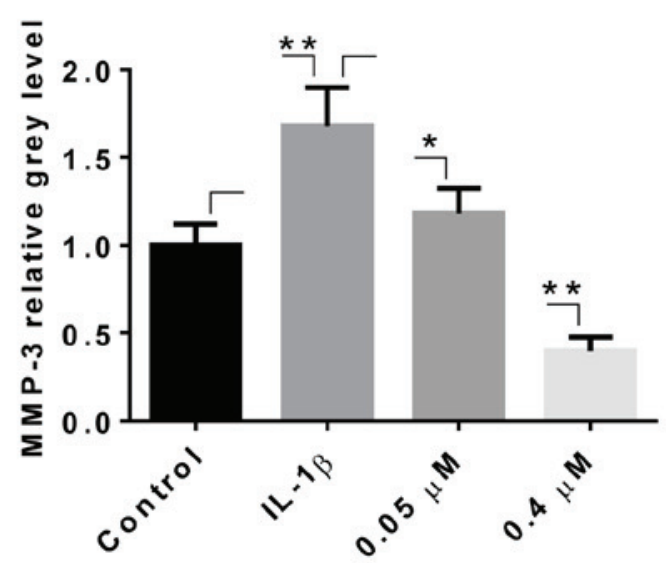

E

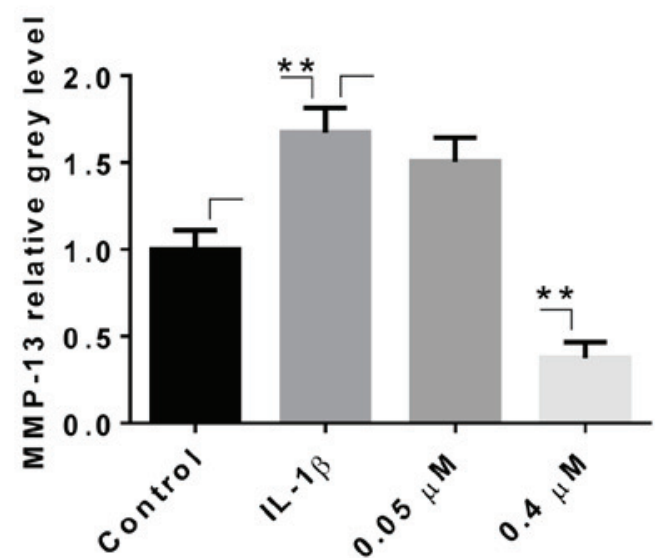

Figure 2. LY inhibits the IL-1 $\beta$-induced expression of MMPs in rat articular chondrocytes. Transcriptional levels of (A) MMP-3 and (B) MMP-13 in chondrocytes treated with $\mathrm{LY}(0,0.05,0.1,0.2$ and $0.4 \mu \mathrm{M})$ and $\mathrm{IL}-1 \beta(10 \mathrm{ng} / \mathrm{ml})$ for $24 \mathrm{~h}$ were analyzed using reverse transcription-quantitative polymerase chain reaction analysis. RNA expression levels were normalized relative to the expression of $\beta$-actin $\left({ }^{*} \mathrm{P}<0.05\right.$ and $\left.{ }^{* *} \mathrm{P}<0.01\right)$. (C) Protein levels of MMP-3 and MMP-13 in chondrocytes treated with LY $(0,0.05$ and $0.4 \mu \mathrm{M})$ and IL-1 $\beta(10 \mathrm{ng} / \mathrm{ml})$ for $48 \mathrm{~h}$ were analyzed using Western blotting. Grey levels of (D) MMP-3 and (E) MMP-13 were quantified and normalized to $\beta$-actin. Data are presented as the mean \pm standard error of the mean $\left(" \mathrm{P}<0.05\right.$ and ${ }^{* *} \mathrm{P}<0.01$ vs. group indicated on graph). LY, lycorine; MMP, matrix metalloproteinase; IL-1 $\beta$, interleukin-1 $\beta$.

were lower, compared with that in the control group, which was not treated with IL-1 $\beta$. The results of the Western blotting were in accordance with those of the RT-qPCR analysis (Fig. 2C-E). The bands corresponding to the MMP proteins in the whole cell lysate showed a significant upregulation of MMPs following treatment with IL-1 $\beta$. This effect was eradicated following treatment with $0.4 \mu \mathrm{M} \mathrm{LY}$. Grey level analysis confirmed this observation.

$L Y$ inhibits IL-1 $\beta$-induced activation of the NF- $\kappa B$ pathway and phosphorylation of JNK in rat articular chondrocytes.
To clarify the underlying mechanisms of the effect of LY in the inhibition of cartilage catabolic activity, the present study investigated key signaling pathways, including the MAPK and NF- $\kappa B$ signaling pathways. The rat articular chondrocytes were incubated with or without LY, and were then treated with IL- $1 \beta$ for $0,5,10,20,30$ and $60 \mathrm{~min}$. As shown in Fig. 3A, the levels of p-JNK in the whole cell lysate were significantly reduced in the groups pre-treated with LY, indicating that LY significantly attenuated the phosphorylation of JNK. The phosphorylation of ERK and P38 were not significantly affected by LY, although delayed 
$\mathbf{A}$

IL-1 $\beta$

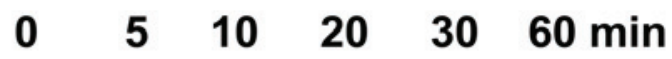

\begin{tabular}{llllll}
0 & 5 & 10 & 20 & 30 & 60 \\
\hline in
\end{tabular}

p-JNK
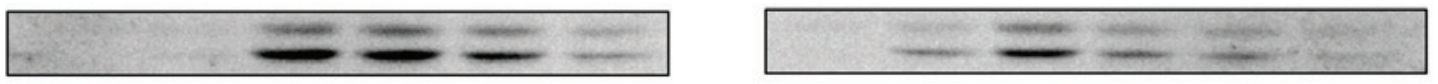

JNK
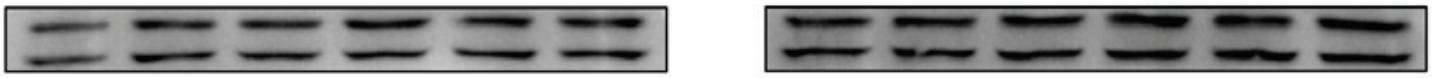

p-ERK
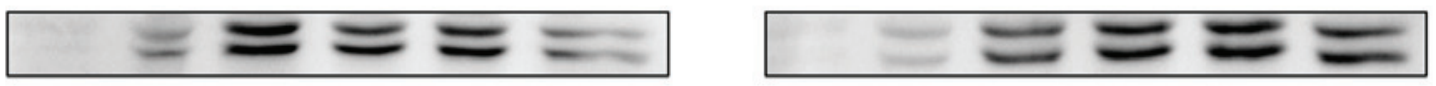

ERK
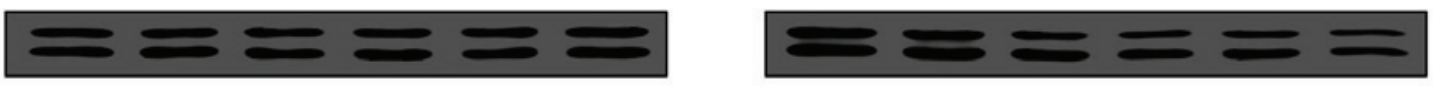

p-P38
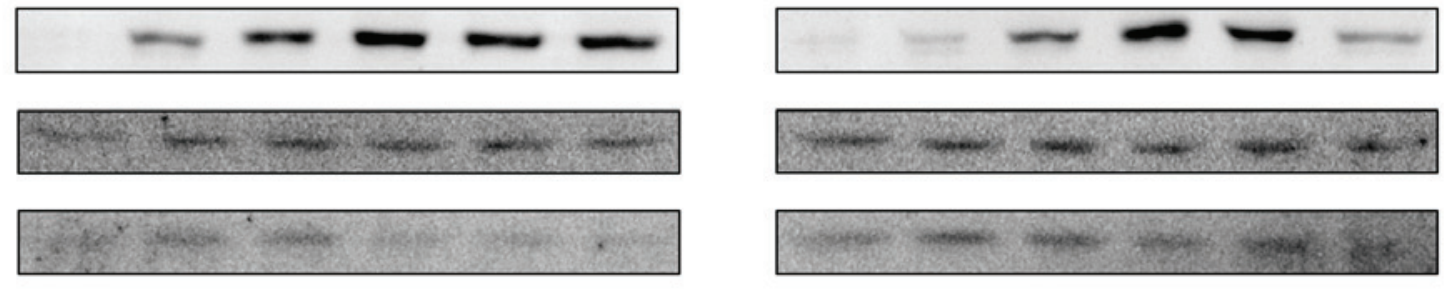

$\beta$-actin
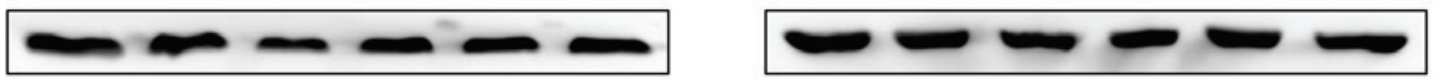

B

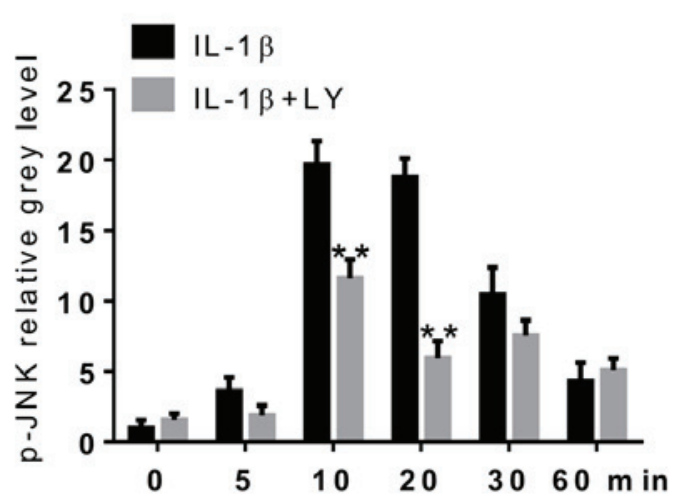

C

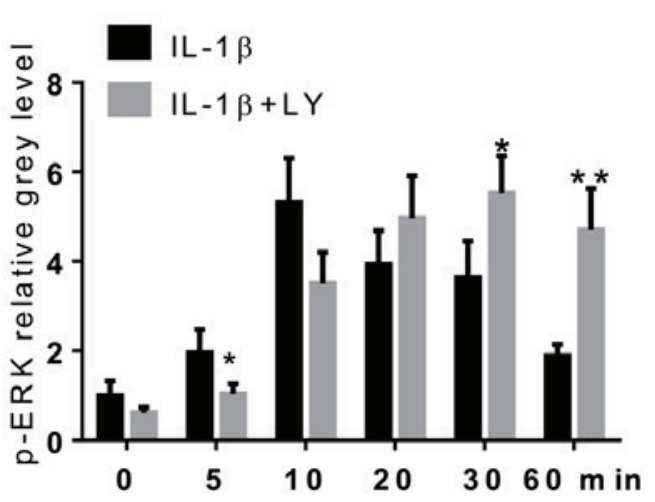

D

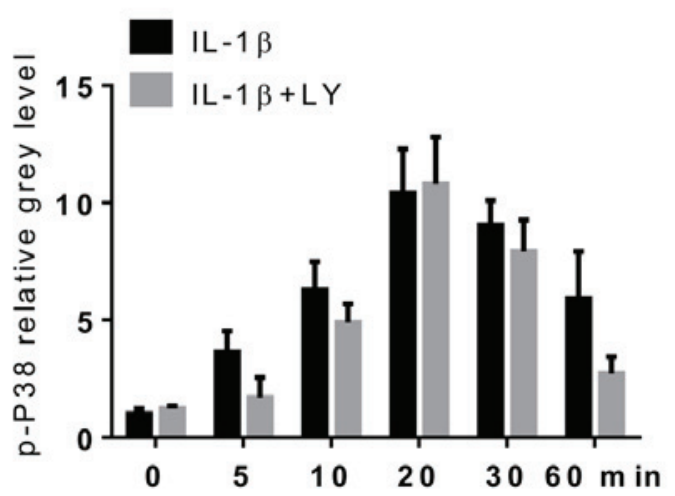

$\mathbf{E}$

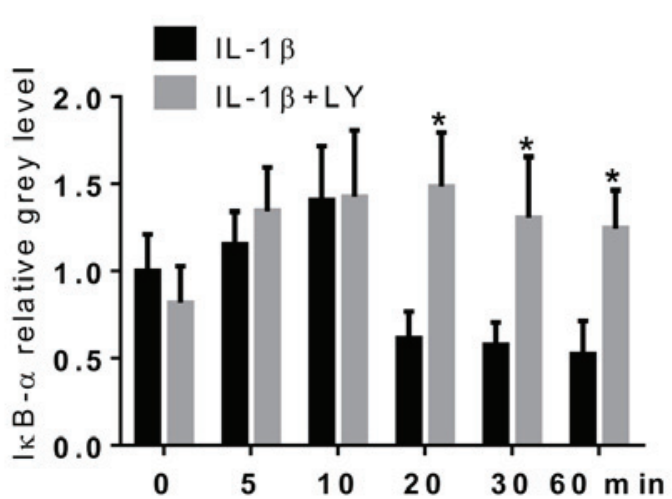

Figure 3. LY inhibits IL-1 3 -induced NF-kB pathway activation and JNK phosphorylation in rat articular chondrocytes. (A) Protein levels of p-JNK, JNK, p-P38, P38, p-ERK, ERK, IKB- $\alpha$ and $\beta$-actin were determined in chondrocytes treated with LY $(0$ or $0.4 \mu \mathrm{M})$ and IL- $1 \beta(10 \mathrm{ng} / \mathrm{ml})$ for $0,5,10,20,30$ or $60 \mathrm{~min}$. :evels were analyzed using Western blotting. Grey levels of (B) p-JNK, (C) p-ERK and (D) p-P38 were quantified and normalized to total JNK, ERK and P38. The grey level of (E) IкB- $\alpha$ was normalized to $\beta$-actin. Data are presented as the mean \pm standard error of the mean ( $" \mathrm{P}<0.05$ and $\left.{ }^{* *} \mathrm{P}<0.01\right)$. LY, lycorine; IL-1 $\beta$,

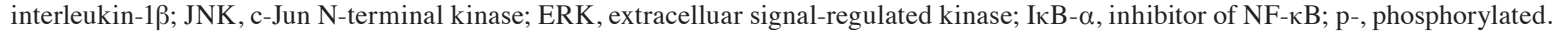

phosphorylation of ERK was observed. The protein levels of I $\mathrm{KB}-\alpha$ were reduced 20,30 and 60 min following IL-1 $\beta$ treatment, which indicated the degradation of IкB- $\alpha$ and activation of the NF- $\kappa \mathrm{B}$ signaling pathway. However, these changes were not observed in the groups pre-treated with LY. The grey level quantitative analysis also confirmed that LY 
A Sham

$100 \times$

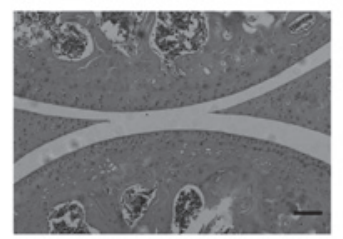

$200 \times$

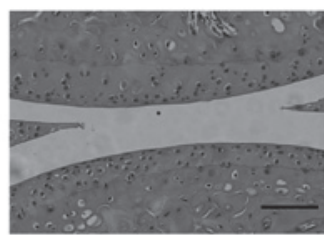

B

$100 \times$

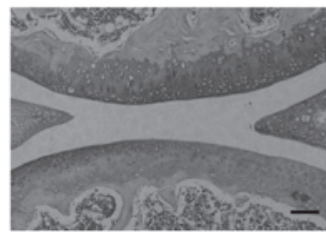

$200 \times$

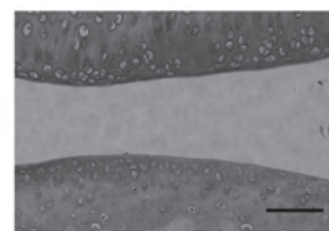

Vehicle
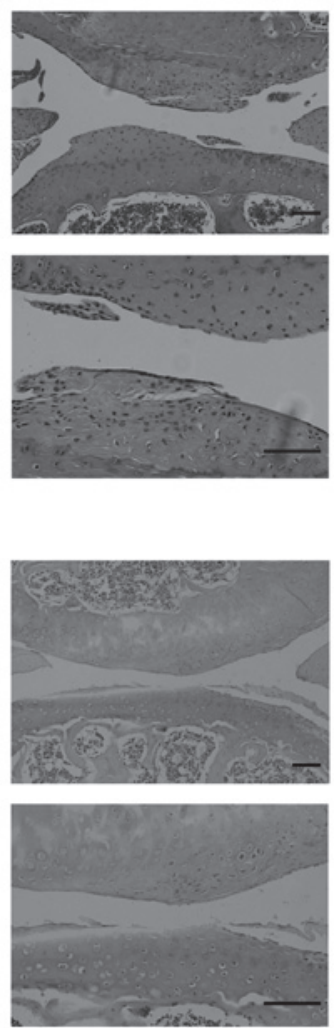

$\mathrm{LY}(2.5 \mathrm{mg} / \mathrm{kg})$
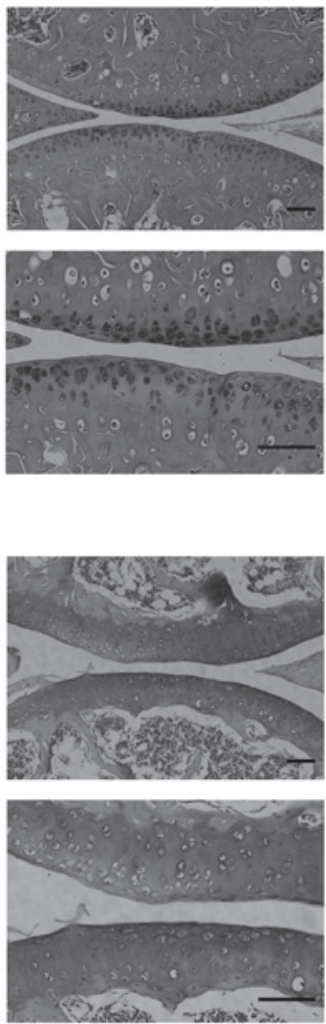

Figure 4. LY effectively protects cartilage in a mouse ACLT model. (A) Hematoxylin and eosin staining of right knee joint sections from the Sham, Vehicle and LY groups (magnification, x100 and x200; scale bar=100 $\mu \mathrm{m}$ ). (B) Safranin O-fast green staining of right knee joint sections from the Sham, Vehicle and LY groups (magnification, x100 and x200; scale bar=100 $\mu \mathrm{m}$ ). Sham, sham surgery + phosphate-buffered saline; Vehicle group, ACLT + phosphate-buffered saline; LY group, ACLT + $2.5 \mathrm{mg} / \mathrm{kg}$ LY. ACLT, anterior cruciate ligament transection; LY, lycorine.

effectively inhibited the IL-1 $\beta$-induced phosphorylation of JNK and activation of the NF-кB pathway (Fig. 3B-E).

LY effectively protects cartilage in a mouse ACLT model. To examine the protective role of LY in cartilage, the present study further examined the effect of LY in a mouse ACLT model. Following treatment with LY for 4 weeks, histological analysis of the joint suggested that LY had a protective effect against ACLT-induced cartilage destruction. H\&E staining showed severe cartilage destruction and fibrillation in the superficial and mid layers of cartilage in the Vehicle group. Matrix discontinuity was also observed in the Vehicle group (Fig. 4A). However, in the LY-treated groups, cartilage destruction was less severe, compared with that in the Vehicle group, although the cartilage surface remained uneven. The SO staining showed that the cartilage matrix was severely reduced in the superficial and deeper layers of cartilage in the Vehicle group, and that the ECM was generally unaffected in the LY-treated groups (Fig. 4B). These histological results in the mouse ACLT model confirmed that LY effectively prevented cartilage degradation.

\section{Discussion}

To the best of our knowledge, the present study is the first to demonstrate that IL-1 $\beta$-induced upregulation of MMP-3 and MMP-13 was significantly suppressed by the administration of the natural alkaloid, LY. In addition, the results showed that this effect was mediated by inhibition of the JNK and NF- $\kappa$ B signaling pathways. The protective effect of LY on articular cartilage was also observed in the mouse ACLT model.

ECM provides tension and absorbs stress in normal articular cartilage, and the dynamic balance between ECM synthesis and degradation is maintained. Biomechanical signaling and inflammatory signaling result in the secretion of IL-1 $\beta$ and TNF- $\alpha$ in chondrocytes, and the recruitment of mononuclear inflammatory cells $(21,22)$. These inflammatory cytokines stimulate the expression of proteases, including MMPs and ADAMTSs. MMPs are the major mediators of cartilage turnover in OA, and are able to degrade all components of the cartilage ECM $(8,23)$. Collagen turnover is almost exclusively mediated by MMPs with collagenolytic ability, including MMP-1 and MMP-13 (8). In articular cartilage, collagenase MMP-13 is the predominant rate-limiting factor in the degradation of the most prevalent type of collagen, type II collagen (8). MMP-3 has 5-10 times more type II collagen cleaving activity, compared with MMP-1. MMP-3 is another important protease and is associated with the degradation of aggrecan, which is the major type of proteoglycan (24). MMP-3 can also cleave other non-collagen cartilage ECM components, including fibronectin, elastin and laminin (8). In the present study, the administration of LY significantly reduced the IL-1 $\beta$-induced expression of MMP-3 and MMP-13 at the mRNA and protein 
levels. Considering the importance of MMP-3 and MMP-13 in $\mathrm{OA}$, it is reasonable to conclude that LY had a protective role in the present in vivo study.

The synthesis of MMPs is predominantly regulated at the transcriptional level by activator protein 1 (AP-1) and $\mathrm{NF}-\kappa \mathrm{B}(25,26)$. AP-1 proteins are $\mathrm{c}-\mathrm{Fos} / \mathrm{c}-\mathrm{Jun}$ heterodimers or c-Jun/c-Jun homodimers. The MMP-1, MMP-3 and MMP-13 promoters contain a key binding site for AP-1 at $-73 \mathrm{bp}$, which is critical for the regulation of their transcriptional activity. The binding activity of AP-1 is regulated by IL- $1 \beta$ and TNF- $\alpha$ through signaling pathways, including the JNK, ERK and P38 pathways (27). JNK can phosphorylate c-Jun to activate the AP-1 DNA-binding complex and promote the expression of MMPs (8). P38 can also activate factors required for the synthesis of AP-1 (8). The stimulation of inflammatory factors results in the phosphorylation and degradation of $\mathrm{I} \kappa \mathrm{B}$, which activates NF- $\kappa \mathrm{B}$ and leads to its translocation into the nucleus. Here, it directly binds to the MMP-1 and MMP-3 promoters, and induces the expression of MMP1 and MMP-3 (26). NF- $\kappa$ B does not bind to the promoter of MMP-13, however, NF- $\kappa \mathrm{B}$ inhibition can lead to the downregulation of MMP-13, which suggests that other mechanisms are involved in regulating the expression of MMP-13 (8). The results of the present study showed that activation of the JNK and NF- $\mathrm{KB}$ signaling pathways by IL-1 $\beta$ was significantly inhibited by LY. Drugs, which are currently used for the treatment of OA, including non-steroidal anti-inflammatory drugs, are unable to stop the destruction of connective tissues. Although substantial effort has been made over several years to identify the inhibitors of MMPs, no effective direct inhibitors have been found; therefore, targeting other molecules in the MAPK or NF- $\kappa$ B pathways may offer a novel method for the treatment for OA. LY is a promising novel drug, which can effectively inhibit the synthesis of MMPs by targeting inflammation-induced pathways.

In conclusion, the present study demonstrated that LY inhibited the IL-1 $\beta$-induced expression of MMP-3 and MMP-13, which are pivotal in the progression of OA. The present study also showed that this effect was mediated by inhibition of the JNK and NF- $\kappa$ B pathways. The results obtained from the mouse ACLT model showed that LY exerted a significant protective effect on cartilage. Taken together, these findings suggested that LY merits consideration as a therapeutic drug in the treatment of OA.

\section{Acknowledgements}

This study was supported by the Chinese National Natural Science Foundation (grant nos. 81271971, 81301587 and 81472064), Natural Science Foundation of Zhejiang Province (grant no. LZ15H060002) the Platform Major Project of Health and Family Planning Commission of Zhejiang province (grant no. 2016145597 and 2015KYA133) and the Project of Education Department of Zhejiang Province (grant no. Y201017108).

\section{References}

1. Mankin HJ, Dorfman H, Lippiello L and Zarins A: Biochemical and metabolic abnormalities in articular cartilage from osteo-arthritic human hips. II. Correlation of morphology with biochemical and metabolic data. J Bone Joint Surg Am 53: 523-537, 1971.
2. Feldmann M: Pathogenesis of arthritis: Recent research progress. Nat Immunol 2: 771-773, 2001.

3. Sandell LJ and Aigner T: Articular cartilage and changes in arthritis. An introduction: Cell biology of osteoarthritis. Arthritis Res 3: 107-113, 2001.

4. Benito MJ, Veale DJ, FitzGerald O, van den Berg WB and Bresnihan B: Synovial tissue inflammation in early and late osteoarthritis. Ann Rheum Dis 64: 1263-1267, 2005.

5. Lee AS, Ellman MB, Yan D, Kroin JS, Cole BJ, van Wijnen AJ and Im HJ: A current review of molecular mechanisms regarding osteoarthritis and pain. Gene 527: 440-447, 2013.

6. Wang X, Wang H, Yang H, Li J, Cai Q, Shapiro IM and Risbud MV: Tumor necrosis factor- $\alpha$ - and interleukin- $1 \beta$-dependent matrix metalloproteinase-3 expression in nucleus pulposus cells requires cooperative signaling via syndecan 4 and mitogen-activated protein kinase-NF- $\kappa \mathrm{B}$ axis: Implications in inflammatory disc disease. Am J Pathol 184: 2560-2572, 2014.

7. Murphy G, Hembry RM, Hughes CE, Fosang AJ and Hardingham TE: Role and regulation of metalloproteinases in connective tissue turnover. Biochem Soc Trans 18: 812-815, 1990.

8. Burrage PS, Mix KS and Brinckerhoff CE: Matrix metalloproteinases: Role in arthritis. Front Biosci 11: 529-543, 2006.

9. Takaishi H, Kimura T, Dalal S, Okada Y and D'Armiento J: Joint diseases and matrix metalloproteinases: A role for MMP-13. Curr Pharm Biotechnol 9: 47-54, 2008.

10. Zeng L, Wang W, Rong XF, Zhong Y, Jia P, Zhou GQ and Li RH: Chondroprotective effects and multi-target mechanisms of Icariin in IL-1 beta-induced human SW 1353 chondrosarcoma cells and a rat osteoarthritis model. Int Immunopharmacol 18: 175-181, 2014.

11. Chen WP, Xiong Y, Shi YX, Hu PF, Bao JP and Wu LD: Astaxanthin reduces matrix metalloproteinase expression in human chondrocytes. Int Immunopharmacol 19: 174-177, 2014.

12. Lee WS, Lim JH, Sung MS, Lee EG, Oh YJ and Yoo WH: Ethyl acetate fraction from Angelica sinensis inhibits IL-1 $\beta$-induced rheumatoid synovial fibroblast proliferation and COX-2, PGE2 and MMPs production. Biol Res 47: 41, 2014.

13. Zupkó I, Réthy B, Hohmann J, Molnár J, Ocsovszki I and Falkay G: Antitumor activity of alkaloids derived from Amaryllidaceae species. In Vivo 23: 41-48, 2009.

14. Cao Z, Yu D, Fu S, Zhang G, Pan Y, Bao M, Tu J, Shang B, Guo P, Yang P and Zhou Q: Lycorine hydrochloride selectively inhibits human ovarian cancer cell proliferation and tumor neovascularization with very low toxicity. Toxicol Lett 218: 174-185, 2013.

15. Li L, Dai HJ, Ye M, Wang SL, Xiao XJ, Zheng J, Chen HY, Luo YH and Liu J: Lycorine induces cell-cycle arrest in the G0/G1 phase in K562 cells via HDAC inhibition. Cancer Cell Int 12: 49, 2012.

16. Liu R, Cao Z, Tu J, Pan Y, Shang B, Zhang G, Bao M, Zhang S, Yang $\mathrm{P}$ and Zhou Q: Lycorine hydrochloride inhibits metastatic melanoma cell-dominant vasculogenic mimicry. Pigment Cell Melanoma Res 25: 630-638, 2012.

17. Kang J, Zhang Y, Cao X, Fan J, Li G, Wang Q, Diao Y, Zhao Z, Luo L and Yin Z: Lycorine inhibits lipopolysaccharide-induced iNOS and COX-2 up-regulation in RAW264.7 cells through suppressing P38 and STATs activation and increases the survival rate of mice after LPS challenge. Int Immunopharmacol 12: 249-256, 2012.

18. National Institutes of Health: Revised guide for the care and use of laboratory animals. NIH GUIDE 25: P.T. 34, 1996.

19. Chen S, Huang Y, Zhou ZJ, Hu ZJ, Wang JY, Xu WB, Fang XQ and Fan SW: Upregulation of tumor necrosis factor $\alpha$ and ADAMTS-5, but not ADAMTS-4, in human intervertebral cartilage endplate with modic changes. Spine (Phila Pa 1976) 39: E817-E825, 2014.

20. Livak KJ and Schmittgen TD: Analysis of relative gene expression data using real-time quantitative PCR and the $2-\Delta \Delta \mathrm{Ct}$ method. Methods 25: 402-408, 2001.

21. Millward-Sadler SJ, Wright MO, Davies LW, Nuki G and Salter DM: Mechanotransduction via integrins and interleukin-4 results in altered aggrecan and matrix metalloproteinase 3 gene expression in normal, but not osteoarthritic, human articular chondrocytes. Arthritis Rheum 43: 2091-2099, 2000.

22. Caron JP, Fernandes JC, Martel-Pelletier J, Tardif G, Mineau F, Geng C and Pelletier JP: Chondroprotective effect of intraarticular injections of interleukin-1 receptor antagonist in experimental osteoarthritis. Suppression of collagenase-1 expression. Arthritis Rheum 39: 1535-1544, 1996.

23. Nagase $\mathrm{H}$ and Woessner JF Jr: Matrix metalloproteinases. J Biol Chem 274: 21491-21494, 1999. 
24. Bonassar LJ, Frank EH, Murray JC, Paguio CG, Moore VL, Lark MW, Sandy JD, Wu JJ, Eyre DR and Grodzinsky AJ: Changes in cartilage composition and physical properties due to stromelysin degradation. Arthritis Rheum 38: 173-183, 1995.

25. Brinckerhoff CE and Matrisian LM: Matrix metalloproteinases: A tail of a frog that became a prince. Nat Rev Mol Cell Biol 3: 207-214, 2002.
26. Barchowsky A, Frleta D and Vincenti MP: Integration of the NF-kappaB and mitogen-activated protein kinase/AP-1 pathways at the collagenase-1 promoter: Divergence of IL-1 and TNF-dependent signal transduction in rabbit primary synovial fibroblasts. Cytokine 12: 1469-1479, 2000.

27. Mengshol JA, Mix KS and Brinckerhoff CE: Matrix metalloproteinases as therapeutic targets in arthritic diseases: Bull's-eye or missing the mark? Arthritis Rheum 46: 13-20, 2002. 